

\title{
Gouverner la voirie urbaine par l'information de l'automobiliste. Une comparaison Lyon-Paris, des années 1920 à nos jours
}

Louis Baldasseroni, Luc Charansonney

\section{- To cite this version:}

Louis Baldasseroni, Luc Charansonney. Gouverner la voirie urbaine par l'information de l'automobiliste. Une comparaison Lyon-Paris, des années 1920 à nos jours. Flux - Cahiers scientifiques internationaux Réseaux et territoires, 2018, Varia, 113 (3), pp.24-40. 10.3917/flux1.113.0024 . hal-01609545

\section{HAL Id: hal-01609545 \\ https: / hal-enpc.archives-ouvertes.fr/hal-01609545}

Submitted on 9 Oct 2018

HAL is a multi-disciplinary open access archive for the deposit and dissemination of scientific research documents, whether they are published or not. The documents may come from teaching and research institutions in France or abroad, or from public or private research centers.
L'archive ouverte pluridisciplinaire HAL, est destinée au dépôt et à la diffusion de documents scientifiques de niveau recherche, publiés ou non, émanant des établissements d'enseignement et de recherche français ou étrangers, des laboratoires publics ou privés. 


\section{GOUVERNER LA VOIRIE URBAINE PAR L'INFORMATION DE L'AUTOMOBILISTE. UNE COMPARAISON LYON-PARIS, DES ANNÉES 1920 À NOS JOURS}

Louis Baldasseroni, Luc Charansonney

Université Paris-Est Marne la Vallée | « Flux »

2018/3 N 113 | pages 24 à 40

ISSN 1154-2721

Article disponible en ligne à l'adresse :

https://www.cairn.info/revue-flux-2018-3-page-24.htm

\section{Pour citer cet article :}

Louis Baldasseroni, Luc Charansonney « Gouverner la voirie urbaine par l'information de l'automobiliste. Une comparaison Lyon-Paris, des années 1920 à nos jours », Flux 2018/3 (N 113), p. 24-40.

DOI 10.3917/flux1.113.0024

Distribution électronique Cairn.info pour Université Paris-Est Marne la Vallée.

(C) Université Paris-Est Marne la Vallée. Tous droits réservés pour tous pays.

La reproduction ou représentation de cet article, notamment par photocopie, n'est autorisée que dans les limites des conditions générales d'utilisation du site ou, le cas échéant, des conditions générales de la licence souscrite par votre établissement. Toute autre reproduction ou représentation, en tout ou partie, sous quelque forme et de quelque manière que ce soit, est interdite sauf accord préalable et écrit de l'éditeur, en dehors des cas prévus par la législation en vigueur en France. Il est précisé que son stockage dans une base de données est également interdit. 


\title{
Gouverner la voirie urbaine par l'information de I'automobiliste Une comparaison Lyon-Paris, des années 1920 à nos jours
}

\author{
Louis Baldasseroni \\ Luc Charansonney
}

$\mathrm{L}$ 'automobiliste est un usager de la voirie arrivé sur le tard. _Objet nouveau, craint au début du XXe siècle, I'automobile s'impose peu à peu sur les chaussées au détriment des autres modes de transport. L'automobiliste, derrière son volant, agit sur la mécanique de son véhicule, en compromis permanent entre son désir de mouvement et un environnement qui le contraint à cohabiter avec d'autres usagers nettement moins rapides, notamment en ville. Les problèmes de confrontation entre l'automobile et les usagers existants deviennent de plus en plus prégnants à mesure que le nombre de véhicules croît, et les autorités y répondent localement par divers règlements dès les années 1900-1910. Dans le contexte de cette première réponse des pouvoirs publics, informer l'automobiliste sur les règles à suivre devient primordial : devant le constat de non-respect de ces règlements, attribué en partie à leur manque de visibilité dans l'espace, les pouvoirs publics réfléchissent à partir des années 1920 à des dispositifs cohérents et adaptés à ces nouveaux usagers à discipliner. II s'agit tout d'abord d'afficher des règles prescriptives, afin d'imposer un sens de circulation, de définir des points d'arrêt et de stationnement. Il s'agit ensuite de matérialiser des normes directionnelles, à destination notamment de l'automobiliste occasionnel, afin de l'orienter vers les lieux de stationnement et les principaux points d'intérêt de la ville. Cette dualité d'objectifs, entre prescription et guidage, est constitutive des systèmes d'information à l'automobiliste, des premiers panneaux de signalisation jusqu'aux GPS et autres applications mobiles actuelles. Cela fonde l'intérêt d'une étude de longue durée de ces dispositifs, pour interroger cette durabilité des principes et des formes dans un contexte d'évolution rapide de la circulation automobile.

Des multiples panneaux en passant par les feux tricolores, I'information de l'automobiliste est au centre de nombreux dispositifs, particulièrement en milieu urbain où les phénomènes de congestion des voies et d'insécurité accroissent les enjeux du contrôle des circulations. En partant de ce constat, cette étude interroge la place de l'information de l'automobiliste dans la gouvernance de la voirie urbaine, rendue complexe par la multiplicité d'activités, de lieux et de gestionnaires. II s'agit de montrer que l'objectif d'informer l'automobiliste prend une place croissante dans les dispositifs de gestion du trafic urbain, qui évoluent en fonction des politiques urbaines et des innovations technologiques. Cependant, la durabilité de ces infrastructures est à souligner, dans la mesure où la plupart survivent aux changements dans les politiques de transports ou de déplacements urbains : I'usage croissant des technologies GPS embarquées ne s'accompagne pas d'un démantèlement des panneaux existants, par exemple. De cet immobilisme résulte une information de l'automobiliste aux dispositifs variés, fruits de près d'un siècle de superposition de strates successives, à la fois techniques et politiques.

L'intérêt d'une étude des dispositifs d'information de l'automobiliste en milieu urbain réside dans les spécificités de celui-ci en termes de partage des voies : I'automobile se diffuse massivement dans un environnement déjà aménagé pour d'autres modes de transport dont les usagers ont aussi des besoins d'information. Cela concerne des acteurs divers, notamment les 
gestionnaires habituels des réseaux de voirie urbaine : les ingénieurs municipaux et ceux des services ordinaires des Ponts et Chaussées, devenus Directions Départementales de l'Équipement en 1967. Les dispositifs d'information à l'automobiliste visibles dans l'espace urbain sont donc le reflet de décisions politiques de gouvernance des transports : on observe par exemple une tension constante entre les objectifs de régulation du trafic et la volonté d'améliorer l'attractivité des espaces urbains pour les automobilistes, particulièrement les touristes.

La complexité des pratiques de circulation sur la voirie urbaine, la variété et l'ancienneté des dispositifs d'information des usagers montrent la pertinence d'analyser ces phénomènes sur le temps long, des premiers panneaux de signalisation aux systèmes électroniques d'information en temps réel. L'étude s'appuie sur une approche comparée de deux grandes villes qui s'inscrivent dans un même contexte de saturation croissante de leurs réseaux viaires par la circulation automobile à partir des années 1910-1920: Lyon et Paris. Ces villes françaises sont déjà bien documentées sur I'histoire des mobilités et des politiques de gestion de la circulation (Flonneau, 2005 ; Gardon, 2009), mais la variété de leurs dispositifs d'information de l'automobiliste incite à en analyser les spécificités locales. À cette démarche comparative de terrains d'étude répond celle d'un échange entre deux champs disciplinaires, l'ingénierie du trafic et l'histoire des formes urbaines, qui s'intéressent ici à un objet commun, la voirie urbaine, avec une vision systémique de la ville, de ses circulations et de ses dispositifs de régulation. À cette vision large de la voirie correspond une pluralité de la notion d'information de l'usager: il s'agit à la fois d'une information géographique (lieux importants, itinéraires) et d'une information réglementaire (affichage des limitations de vitesse, des interdictions,...), qu'il est important d'articuler. Constituée au départ de dispositifs ponctuels adaptés à des situations spécifiques, l'information donnée à l'automobiliste connaît un mouvement de diversification, d'extension dans les villes. Elle devient un instrument privilégié de politiques globales de gestion du trafic pouvant inclure d'autres modes de transport.

Dans ce contexte d'extension des dispositifs d'information à l'automobiliste, Paris et Lyon suivent globalement les mêmes dynamiques d'évolution des divers enjeux de ces politiques: sécurité, gain de temps, lutte contre la pollution,... Cette proximité chronologique globale cache cependant des décalages ponctuels entre les deux villes selon les dispositifs, reflet d'une autonomie locale forte : malgré la précocité des politiques de gestion de la circulation automobile (Flonneau, 2003), Paris ne semble pas constituer un modèle pour Lyon. Ces différences s'observent davantage dans la nature des dispositifs et la chronologie de leur mise en œuvre que dans les orientations politiques des gestionnaires, guidées par leurs imaginaires de I'automobiliste : cela conduit à distinguer trois «strates » de dispositifs qui répondent à des ruptures historiques et politiques dans ces représentations.

La première strate, qui correspond aux années 1920 à 1940, se manifeste dans le guidage de l'automobiliste occasionnel, vers sa destination. Pour le gestionnaire, I'automobiliste est encore un individu qu'il faut guider « individuellement ». Cela se traduit techniquement par une signalétique statique de prescription et d'indication.

La deuxième strate débute après la Seconde Guerre mondiale et se poursuit jusque dans les années 1970 : I'automobiliste en tant qu'individu devient une composante d'un flux de plus en plus massif. À la hausse inéluctable de la demande automobile répond une politique de l'offre, à la fois par la création d'infrastructures routières dédiées, et par l'adaptation de I'existant. Techniquement, ce sont les signaux lumineux tricolores, dits aussi feux de circulation, qui occupent une place capitale en zone urbaine, et permettent une régulation des flux par le biais de systèmes coordonnés et centralisés.

Enfin, la troisième strate s'observe à partir des années 1980. La diversité des modes de transport sur le réseau viaire est prise en compte, et la diffusion de la télématique permet une individualisation du suivi des déplacements, I'usager devenant en retour lui-même source d'information, les données pouvant s'échanger sur divers réseaux.

\section{ANNÉES 1920 - ANNÉES 1940 : INFORMER L'AUTOMOBILISTE SUR LA RÉGLEMENTATION, LE GUIDER VERS SA DESTINATION}

La question de l'information des usagers se pose avec une particulière acuité à partir des années 1920, lorsque la circulation automobile commence à poser des problèmes d'encombrement et de sécurité, pour lesquels le manque d'informations sur les itinéraires ou les conditions de circulation est souvent mis en cause. L'automobile constitue alors une part relativement faible des transports de surface. Le gestionnaire est en mesure de considérer l'automobiliste comme un individu, auteur d'un déplacement occasionnel, qu'il faut guider à travers une ville 
dont le réseau viaire n'est guère adapté. II faut d'abord I'informer des règlements en vigueur concernant le stationnement et les premiers sens uniques de circulation, puis lui indiquer comment se rendre vers sa destination, ou encore susciter son intérêt de visiteur. L'outil technique majeur du gestionnaire est alors le panneau statique, dont le déploiement s'avère complexe dans les deux villes, aux enjeux légèrement différents.
L'automobiliste occasionnel : informer des règlements, garantir la sécurité

L'implantation de panneaux de signalisation ne date pas des débuts de l'automobile (Figure 1). Sa nécessité est néanmoins de plus en plus affirmée à partir des années 1920, notamment pour les automobilistes (Duhamel-Herz, Nouvier, 1998). Cette information de l'usager des rues se fait principalement par des

Figure 1. Poteau-indicateur avenue des Champs-Élysées : " Avenue interdite aux voitures publiques attelées de plus de deux chevaux et aux voitures de roulage et de transport suspendues ou non suspendues »

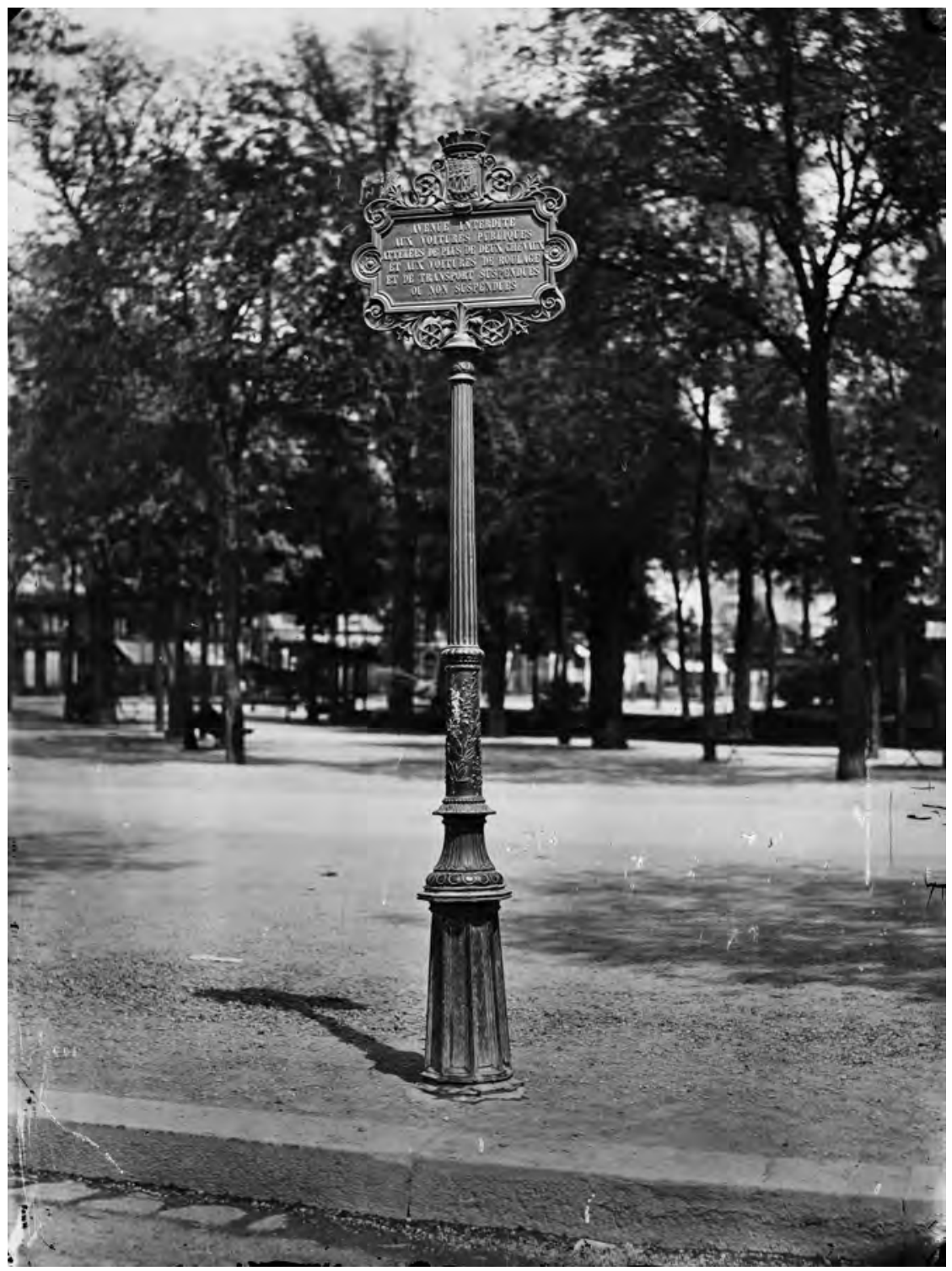

Source : Charles Marville (photographe), vers 1870. (c) Charles Marville / Bibliothèque historique de la Ville de Paris / Roger-Viollet. 
panneaux de signalisation indiquant des lieux et voies importants, pratique qui fait l'objet de réflexions nouvelles à partir des années 1920, pour indiquer les sens uniques établis dans de nombreuses villes françaises (dont Lyon et Paris), les points d'arrêt de réseaux de transports en commun en plein développement, ou encore les lieux et itinéraires importants pour orienter les visiteurs étrangers.

Avec le développement du tourisme automobile, Paris et Lyon, comme la plupart des villes européennes, reçoivent de plus en plus de personnes extérieures à la ville qui n'en connaissent pas forcément les principes d'organisation spatiale ni les règles de circulation. Ces règles connaissent d'ailleurs à partir des années 1920 un phénomène d'accroissement et de complexification, avec par exemple les premiers règlements de la circulation (1910 à Paris, 1926 à Lyon) ou encore les sens uniques, qui peuvent désorienter les automobilistes extérieurs et les amener à commettre des infractions dommageables pour la circulation locale et le plaisir de leur séjour. L'intérêt pour la signalisation automobile vient aussi des associations d'usagers, principalement l'Automobile-Club et le Touring-Club de France, ainsi que des industriels du secteur comme Michelin ou Citroën, qui s'impliquent dans le développement des panneaux de signalisation en fournissant leurs propres panneaux à des gestionnaires et des municipalités aux budgets restreints, panneaux déployés tant en zone rurale qu'urbaine (Faugier, 2009). Ainsi, le premier plan de signalisation automobile lyonnais est proposé par la société Citroën, qui prévoit la pose de quatre-vingt-dix-sept plaques émaillées sur des poteaux d'éclairage municipal pour indiquer le centre-ville et la direction des principales grandes villes accessibles depuis les grands axes lyonnais. Ce plan a pour but de répondre au "problème de signalisation de Lyon » posé par le président du Syndicat d'Initiative, examiné lors d'une réunion qui rassemble les ingénieurs des services de l'État, du département et de la ville, mais aussi des représentants du Touring-Club de France et de l'Automobile-Club du Rhône : tous s'accordent sur la nécessité de mieux guider l'automobiliste, mais les budgets publics consacrés à la signalisation sont plutôt faibles (1). Il est question que le coût de la pose des plaques soit partagé entre le service des Ponts et Chaussées, qui gère les routes nationales sur lesquelles ces panneaux seront posés, et la Chambre d'Industrie Touristique, rattachée à la Chambre de Commerce et d'Industrie de Lyon, preuve que les enjeux touristiques sont ici plus importants que ceux de la gestion des circulations sur la voirie.
Les principaux enjeux de l'information des automobilistes sont mis en évidence et les premières actions sur ce plan se concrétisent au début des années 1920. Ainsi, la réglementation de la circulation automobile, de plus en plus précise, fait l'objet d'une campagne de diffusion large : à Lyon, le premier Règlement général de la circulation de 1926 est imprimé à plusieurs centaines d'exemplaires sous forme $d^{\prime}$ 'un petit guide de trente-six pages en format de poche qui compile les règles générales, toutes les dispositions particulières concernant certaines rues et, par la suite, tous les arrêtés municipaux rectificatifs (2). Outre la connaissance de la réglementation, l'enjeu principal de l'information des automobilistes en milieu urbain dans les années 1930 réside dans l'amélioration de la sécurité, notamment pour éviter les accidents entre automobiles et piétons, les plus fréquents et souvent mortels : c'est par exemple dans ce but qu'est inauguré en 1912 le premier kiosque-signal à Paris, au croisement entre le boulevard Montmartre et la rue du Faubourg-Montmartre (Flonneau, 2005, p. 78-79). À la composante de sécurité des circulations, s'ajoute à partir des années 1930 la congestion, qui touche certes le centre de Lyon et de Paris depuis longtemps, mais prend une importance nouvelle avec le développement exponentiel du trafic automobile.

\section{La hausse du trafic, les premières congestions : schémas globaux de signalisation et innovations techniques}

L'information aux automobilistes devient alors plus globale, avec la mise en œuvre de politiques à l'échelle de la ville entière : il apparaît d'une importance croissante de signaler aux automobilistes occasionnels ou extérieurs à la ville les emplacements disponibles pour le stationnement, ainsi que les principaux lieux touristiques, administratifs et d'affaires. Cela se concrétise par la mise en place de plans de signalisation, qui se fait de manière parallèle à Paris et à Lyon au début des années 1930. Cette forte similitude des premiers dispositifs d'information dans les deux villes s'explique en grande partie par l'influence des associations d'usagers comme I'Automobile-Club et de firmes nationales. D'autre part, ces premiers plans concernent surtout des routes nationales en raison de leurs forts enjeux touristiques, ce qui met en avant la compétence des ingénieurs des Ponts-et-Chaussées, favorables aussi à l'unicité des solutions.

Ces plans d'action pour l'automobile à l'échelle de la ville s'inscrivent dans un moment de basculement des politiques 
de transport en faveur de l'automobile et au détriment des réseaux ferrés sur voirie. Paris, avant Lyon et même bon nombre de grandes agglomérations européennes, entame ainsi le démantèlement systématique de son réseau de tramway entre 1927 et 1938, en commençant par la zone centrale et en terminant par la banlieue. Du côté lyonnais, les premiers projets de conversion de lignes de tramways en autobus datent de 1926 et des tramways ont parcouru la ville jusqu'en 1957 (Gardon, 2011) : cette plus grande progressivité est due à la moindre urgence du problème de congestion par rapport à Paris, mais aussi aux difficultés budgétaires de la compagnie de transport locale dans les années 1930 et à l'obsolescence du réseau à la fin de la Seconde guerre mondiale. La congestion demeure l'un des principaux arguments avancés pour ces campagnes de report sur autobus de ces lignes de transport en commun en lien avec une pression importante des constructeurs automobiles, exploitant notamment des lignes d'autobus parallèles aux lignes de tramway les plus rentables (Bannat, 1995).

Ainsi, le premier plan de signalisation de Lyon, soumis à la municipalité en 1931 et concrétisé en 1934, affiche trois buts pour l'automobiliste: premièrement, aider l'automobiliste terminant son séjour à sortir de la ville ; deuxièmement, aider celui qui entre en ville à "trouver facilement la place Bellecour, constituant le point le plus central de Lyon »; troisièmement, «faciliter l'écoulement des automobilistes ne voulant pas s'arrêter à Lyon en leur faisant éviter l'encombrement du point central » (3). La tension entre les impératifs d'accueil des touristes et d'action contre les encombrements est ici clairement visible, avec semble-t-il ici une priorité donnée aux enjeux touristiques d'accessibilité du centre-ville, tension probablement due aux enjeux importants du trafic de transit dans la capitale des Gaules. À Paris, il s'agit davantage de traiter les encombrements, bien plus importants et considérés comme plus urgents qu'à Lyon. Pour cela, des innovations techniques dans la signalisation sont très tôt mobilisées, avec dès 1923 l'équipement de plusieurs carrefours centraux en signalisation lumineuse à Paris et leur généralisation rapide (Flonneau, 2005, p. 114), alors qu'il faut attendre 1937 à Lyon pour voir apparaitre un feu tricolore, sans généralisation avant les années 1950 (Gardon, 2009, p. 145).

Ce premier temps de l'information à l'automobiliste en milieu urbain est donc marqué par la mise en place de dispositifs simples pour donner des informations géographiques et réglementaires utiles à l'insertion de ce nouveau mode de transport dans la vie urbaine. Sur ce plan, Paris et Lyon suivent les mêmes dynamiques, de manière plus progressive dans la seconde. Cependant, les jalons des politiques d'information à l'automobiliste encore en vigueur aujourd'hui sont posés : élargissement à l'échelle de la ville entière, innovations technologiques mises au service de la circulation automobile. Une vraie rupture s'opère par la suite, à partir des années 1950, dans la mesure où l'automobiliste est de moins en moins pris en compte dans son individualité par les gestionnaires de la voirie.

\section{ANNÉES 1950 - ANNÉES 1970 : RÉGULER LE FLUX AUTOMOBILE}

La hausse du trafic automobile s'interrompt avec la Seconde Guerre mondiale et les réquisitions tant des autobus que des véhicules particuliers qui s'ensuivent. À Paris comme à Lyon, la reprise économique à la fin des années 1940 entrâne des problèmes de congestion toujours plus nombreux. L'automobiliste est alors considéré comme partie prenante d'un flux par des gestionnaires de la voirie influencés par les pratiques américaines $\mathrm{d}^{\prime}$ « ingénierie du trafic » (4). La mission de cette dernière est alors d'orienter et répartir ce flux sur le réseau viaire existant, tout en participant à la planification de nouvelles infrastructures. La politique de l'offre, prégnante jusqu'au début des années 1970, s'infléchit ensuite progressivement, au profit d'une meilleure information du gestionnaire, puis de l'usager, afin de faire le meilleur usage possible des infrastructures existantes. Dans ce contexte, les gestionnaires des voiries parisienne et lyonnaise mettent en œuvre les mêmes solutions, avec quelques années de décalage pour Lyon, dont les flux automobiles sont moins nombreux.

\section{Répondre à la congestion par l'offre : I'information comme outil de régulation du trafic pour le gestionnaire}

À partir des années 1930 et surtout de l'après Seconde Guerre mondiale, les aménagements des voies urbaines sont davantage pensés pour l'automobile que pour les autres modes de transport, tant à Paris (Flonneau, 2005) qu'à Lyon (Gardon, 2011). Cela conduit les aménageurs à appliquer aux rues des principes et des dispositifs venus de l'aménagement des routes : ségrégation des trafics, élargissement et goudronnage des voies au détriment des trottoirs, entre autres. L'automobiliste n'est cependant pas au centre des dispositifs d'information en tant qu'individu, mais en tant que composante d'un flux automobile sur une voie à fluidifier. Cela se voit par exemple sur la 
Figure 2. Flèche d'anticipation portant l'indication

" Maine seulement », au droit du carrefour Maine-Montparnasse-Vaugirard, années 1950

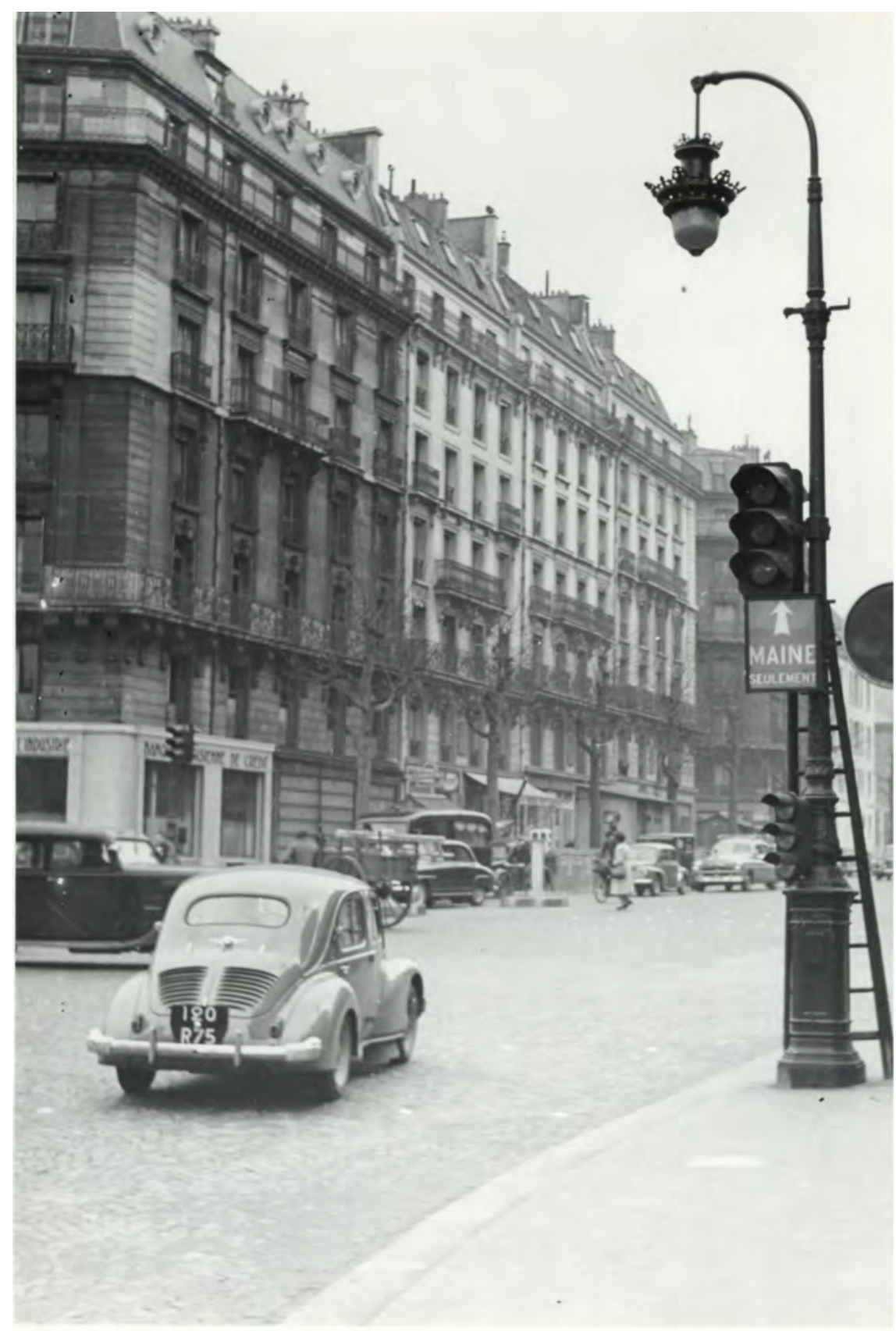

Source : Archives du LER, Paris

Figure 2, où une flèche lumineuse $\mathrm{d}^{\prime}$ anticipation est adjointe au signal tricolore, permettant d'augmenter la capacité automobile de traversée du carrefour en autorisant un mouvement (ici, vers l'avenue du Maine) même lorsque le signal est au rouge, au détriment donc des traversées piétonnes. Ces conceptions, en grande partie influencées par les traffic engineers américains 
et adoptées par le corps des ingénieurs des Ponts-et-Chaussées en France (Dupuy, 1975), prennent peu en compte la diversité des usages et des usagers et conduisent à des choix d'information pour des objectifs très précis et assez éloignés des préoccupations individuelles des automobilistes, jusqu'aux années 1990.

Si la sécurité reste une préoccupation importante tout au long de la période étudiée, les dispositifs d'information des automobilistes ne sont plus au centre des politiques de "sécurité routière ", qui reposent davantage sur des équipements concernant les piétons en ville : passages cloutés, barrières de protection, actions éducatives et campagnes de presse. À partir des années 1950, l'impératif de lutte contre la congestion semble prendre le pas sur les autres objectifs de l'information aux automobilistes, à Lyon comme à Paris. Cela s'explique par la prise en compte de la congestion comme un objet d'étude à part entière après-guerre, au sein de l'ingénierie du trafic (Barles, Guillerme, 1998) : d'un phénomène observé ponctuellement dans les années 1930 dans le cadre des premières campagnes métrologiques «scientifiques » menées sur la circulation (5), la congestion devient prégnante dès les années 1940 aux États-Unis (Greenshields, 1947), puis au début de la décennie suivante en Europe. Le développement de l'information à l'automobiliste est à mettre en parallèle avec celui des études de trafic qui se généralisent à partir des années 1950 avec des méthodes et du matériel importés des États-Unis (Dupuy, 1975 ; Debizet, 2011). Cela s'inscrit aussi dans la tendance à la précision croissante des comptages de circulation et à la focalisation sur les déplacements plutôt que les modes euxmêmes : les axes à jalonner correspondent surtout aux axes de grande circulation à caractéristiques routières et aux itinéraires les plus sujets à la congestion par leur caractère structurant.

L'information des automobilistes, si elle se pense au départ comme moyen d'orientation individuelle, évolue après la guerre vers une gestion plus globale des flux par la régulation du trafic. Par la suite, on observe une "globalisation » des initiatives d'information aux automobilistes, tant à Lyon qu'à Paris : les dispositifs vus précédemment se diversifient et s'étendent à la ville entière, avec une réflexion sur leur utilité qui appuie la naissance des premières politiques intégrées de "régulation du trafic » (l'expression apparaît dans les années 1950). Cette orientation repose sur deux piliers : le premier est l'infrastructure, par la définition de programmes d'autoroutes et voies express urbaines; le deuxième est le déploiement de signaux tricolores à feux sur les artères principales et la coordination des carrefours entre eux par le biais d'ondes vertes dès la fin des années 1940 et la mise en place de postes centraux de coordination pour certains secteurs (Cité pour le centre de Paris, conçu en 1948, suivi dans les années 1950 par Maillot, Pasquier et Bergère), première étape d'une régulation « dynamique » des flux. On assiste là à une première mise en scène de ces "salles de contrôle", équipées des premiers synoptiques, comme on peut le voir sur la Figure 3 avec celle de la porte Maillot. Là encore, Lyon suit cette tendance de manière beaucoup plus progressive, avec une politique d'équipement massif des carrefours en feux dans les années 1960 et leur coordination au début des années 1970 (Gardon, 2011, p. 64-65). Cela $s^{\prime}$ explique sans doute par les flux automobiles beaucoup moins importants à Lyon, mais aussi par la politique d'infrastructures du maire Louis Pradel (1958-1976), qui privilégie des dispositifs permettant d'éviter les feux, comme des trémies et une autoroute urbaine traversant la ville (Montès, 2003, p. 75-77).

La place croissante prise par ces dispositifs d'information dans l'espace et dans la politique des transports est de fait une réponse aux problèmes grandissants posés par l'augmentation du trafic. Cependant, le coup d'arrêt donné à la création d'infrastructures routières en milieu urbain après le choc pétrolier de 1973 entraîne des mutations importantes dans la gouvernance des transports, à Paris comme à Lyon.

\section{Vers des politiques coordonnées, multimodales et des informations qualitatives}

L'élargissement des dispositifs d'information aux automobilistes s'observe aussi en ce qui concerne les pouvoirs publics, qui aspirent à une régulation coordonnée entre voirie urbaine et réseaux structurants gérés par l'État. Ce dernier organise l'information dès 1966-1968 avec la Gendarmerie, qui aboutit à la création du Centre National d'Information Routière (CNIR) en 1969 et, entre 1972 et 1980, à des Centres Régionaux $d^{\prime}$ Information et de Coordination Routière (CRICR), dont un dédié à l'île-de-France (Rosenthal, 2006). Les Directions Départementales de l'Équipement (DDE) assurent le lien entre ces centres d'information et les acteurs locaux. À l'échelle des voiries gérées par les agglomérations et les départements, dont l'étendue s'accroît au fur et à mesure des décentralisations successives, la détermination des outils de l'information trafic donnée aux automobilistes et des itinéraires ciblés sont le fruit de compromis locaux, tant à Lyon qu'à Paris. Dans Paris 


\section{Figure 3. Image du Poste Maillot (vers 1956), Laboratoire des Équipements de la Rue}

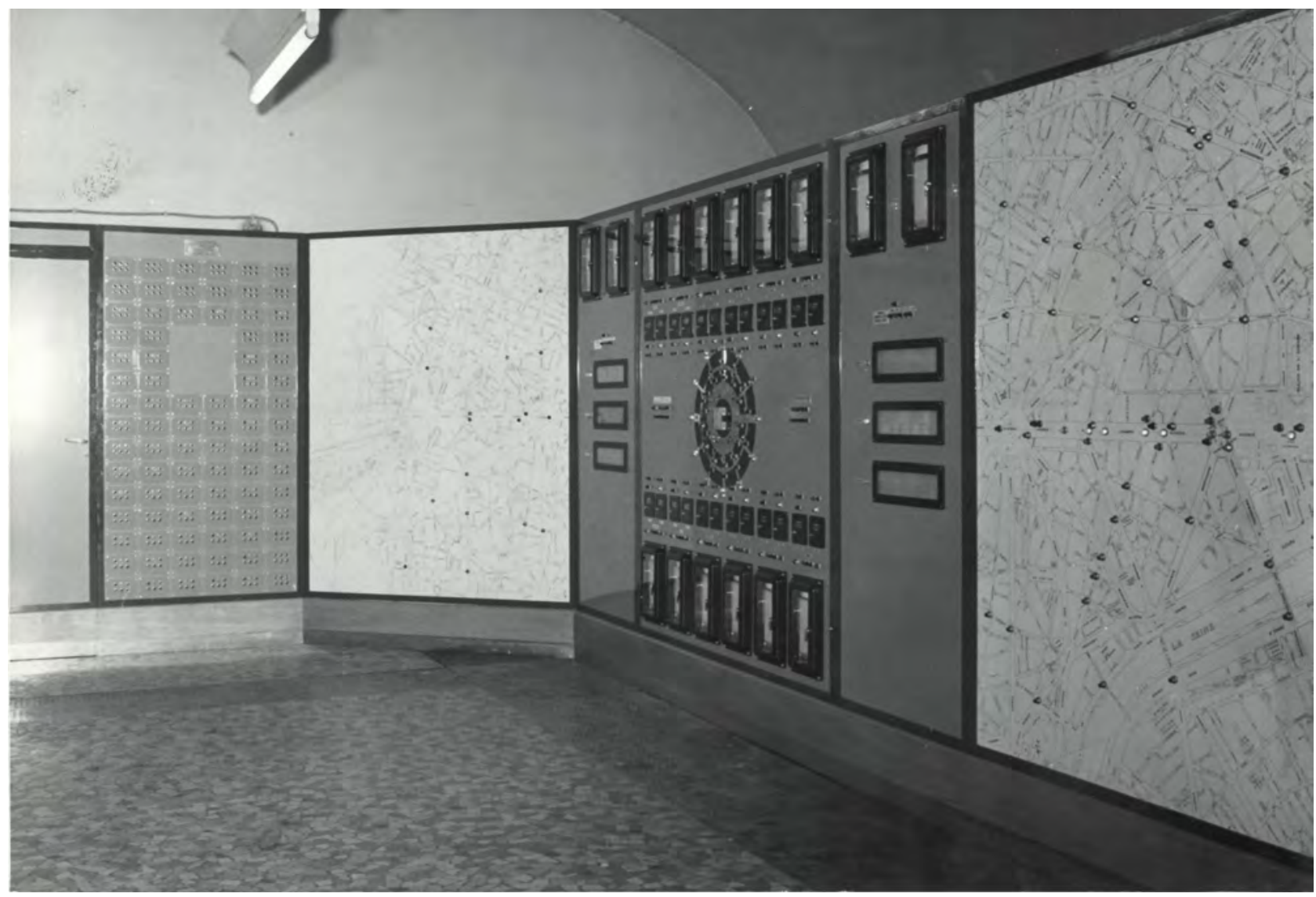

Source: Photographie reproduite par les auteurs avec l'aimable autorisation du regretté Pierre Leroy, responsable de la division Circulation et signalisation du Laboratoire de 2000 à 2017.

intra-muros, un réseau principal de voirie existe déjà depuis les années 1950 et forme la base des plans de circulation comme celui de 1976. À partir de 1976, suite au plan de jalonnement qui en découle, I'administration implante progressivement des mâts directionnels éclairés, conçus et fabriqués par la société JCDecaux. Ces mâts « permettent le déplacement et l'orientation des automobilistes sur la base d'un maillage de l'espace urbain faisant apparaître des nœuds routiers remarquables » selon le schéma directeur de signalisation de 1985 (6). Ce déploiement intervient alors qu'est élu en 1977 le premier maire de Paris depuis 1871, Jacques Chirac.

Les deux villes prêtent également une attention particulière à leur boulevard périphérique, justifiée par leurs enjeux particulièrement importants de redistribution des flux. La construction du boulevard Périphérique parisien, dont le premier tronçon ouvre en 1960, introduit à la fois un nouveau type de voie pour la ville (la voie rapide urbaine, qualifiée ici de "boulevard ») et de nouvelles signalisations aux portes de Paris. Avec l'achèvement du boulevard Périphérique en 1973, le réseau viaire de la Ville est alors scindé en deux parties aux caractéristiques bien différentes : d'un côté le boulevard Périphérique, doté de mesures spécifiques d'exploitation apparentées au domaine autoroutier, malgré son statut de voie communale, et de l'autre le réseau intra-muros, dont l'armature est le réseau principal de voirie, progressivement supervisé par un système de pilotage centralisé de la signalisation lumineuse tricolore. À Lyon, le périphérique est beaucoup plus ancien puisqu'il est inauguré en tant que boulevard Laurent Bonnevay en 1938, avec des caractéristiques " urbaines » (croisements à niveau). Il est donc exclu des premiers plans de signalisation du début des années 
1930. Il est par la suite réaménagé avec des caractéristiques autoroutières à partir de 1958 (échangeurs dénivelés, chaussées unidirectionnelles séparées comptant trois voies et plus chacune) et requalifié en route nationale (Gardon, 2011, p. 6063). Il devient alors un axe prioritaire à jalonner: sa nouvelle signalisation, calquée sur le modèle du périphérique parisien, contribue à le distinguer de la voirie urbaine pour en faire un véritable axe routier consacré au contournement de la ville et à l'irrigation de ses entrées.

Par ailleurs, dans le cadre de la mise en œuvre des politiques d'encouragement à l'intermodalité décidées à la fin des années 1970 dans les grandes villes françaises, l'interconnexion des systèmes de gestion des différents modes de déplacement devient progressivement une préoccupation importante. Les gestionnaires de la voirie sont conduits à prendre en compte les autres modes de transport, y compris dans les programmes de signalisation aux automobilistes. Cela sous-entend l'interconnexion des systèmes entre eux, car à chaque réseau de capteurs correspond un gestionnaire de l'infrastructure où il est implanté, propriétaire du réseau et du système en aval desdits capteurs. À Paris, le jalonnement de 1976, complété en 1985 par le Schéma directeur de la signalisation d'indication (7), ne s'intéresse plus uniquement à la signalisation automobile mais aussi à standardiser la signalétique piétonne, vers des pôles d'intérêt d'un " accès parfois malaisé pour les piétons, notamment à partir des points d'arrêt des transports en commun ou des parcs de stationnement pour automobiles ». Ce schéma traduit la préoccupation grandissante pour la multimodalité, ainsi qu'un recul de l'automobile dans les priorités politiques : il « s'adresse principalement aux usagers piétons avec, lorsque cela s'avérera nécessaire, un complément d'indications pour les automobilistes ». Ainsi, l'ensemble des points d'intérêt de la ville n'est plus nécessairement considéré comme directement accessible en automobile. Ce n'est par contre pas le cas à Lyon, dont le plan de jalonnement de l'agglomération de 1977 reste dédié à l'automobile, sans prise en compte des autres modes de transport: cela peut s'expliquer par la priorité donnée à l'intégration d'un système centralisé de commande des feux, déjà opérée à Paris depuis la fin des années 1940 (par zone) mais fait nouveau à Lyon. Ce plan, étudié par le CETE (Centre d'Études Techniques de l'Équipement), associe un plan de signalisation des axes principaux et des carrefours importants à un plan de régulation du trafic avec un système centralisé de commande des feux sur les grands axes (8). II faut attendre le programme lyonnais LEADER de 1992 pour intégrer d'autres modes de transport dans les schémas de signalisation, par la coordination des feux en fonction du passage des bus (9). Ce dispositif reste cependant conçu en priorité pour mieux gérer les flux automobiles. À Lyon, la signalétique piétonne s'observe essentiellement dans les premières zones et rues piétonnes e à partir de la fin des années 1970, avant une généralisation dans les années 1990.

Ces dispositifs s'inscrivent dans un contexte d'évolution des méthodes de régulation du trafic, avec une prise en compte des déplacements plus qualitative, à partir des années 1960 . Les gestionnaires de la voirie prennent conscience que cette gestion quantitative des flux automobiles ne parvient pas à enrayer l'encombrement toujours croissant des centres-villes. Ils se tournent alors vers des méthodes plus qualitatives, en complément des méthodes traditionnelles d'analyse du trafic : cela s'observe par exemple avec les premières EMD (Enquêtes Ménages-Déplacements), initiées en 1960, qui comportent une étude de déplacements dont les résultats sont repris pour définir de nouvelles politiques de transport.

Les années 1950 à 1970 constituent donc un moment de rupture dans l'information aux automobilistes, tant à Paris qu'à Lyon : la régulation du trafic devient la priorité absolue pour lutter contre la congestion, ce qui conduit rapidement à des politiques de coordination entre les différentes collectivités gestionnaires et entre différents modes de transport. La tendance à l'orientation de flux plutôt qu'à des indications sur les modes et les motifs des déplacements individuels semble s'infléchir dès les années 1970, avec l'abandon progressif, en ville, des programmes d'infrastructures de circulation exclusivement dédiées à l'automobile et le développement de l'informatique.

\section{Des anNées 1980 À nos JOURS : LA RÉVOLUTION DU GUIDAGE DYNAMIQUE DES DÉPLACEMENTS}

À partir de la fin des années 1970, la gouvernance de la voirie urbaine s'appuie sur d'importantes innovations techniques, qui permettent au gestionnaire d'élaborer des systèmes centralisés de régulation et d'information sur le réseau dont il a la charge. Cela accompagne la volonté des pouvoirs publics de fournir un guidage et une information dynamiques aux automobilistes, tant en amont que pendant leur déplacement. Dans ce contexte, les Parisiens et les Lyonnais conçoivent des systèmes 
d'information bien différents, mais avec le même objectif d'une information de plus en plus adaptée aux conditions de trafic en temps réel. Néanmoins, le gestionnaire, initialement au centre des dispositifs d'information va, à partir des années 1990, du fait de la démocratisation de la télématique embarquée, voir son monopole peu à peu amputé par de nouveaux acteurs.

\section{L'âge du système central, le gestionnaire au centre de la chaîne de l'information}

À partir des années 1980, le gestionnaire peut s'adresser plus directement à I'automobiliste en cours de déplacement par I'intermédiaire des Panneau à Message Variable (PMV), qui se multiplient dans les grandes villes et leurs régions. Le passage à une information trafic dynamique, et non plus seulement statique, est rendu possible par le développement de nouvelles technologies. Le PMV est alors vu comme le seul moyen pouvant être déployé à grande échelle pour fournir une information " en cours de trajet » et continue, en complément de la couverture nationale et locale de l'information trafic assurée par les radios FM (RMC, FIP...). Si les techniciens parisiens et lyonnais font rapidement usage des PMV, I'ampleur du déploiement est plus réduite à Lyon, ce qui correspond à une volonté de dissociation plus forte entre axes de transit et axes urbains. Ainsi, le dispositif parisien est axé sur la coordination entre voirie locale et grands axes de circulation, ce qui amène à équiper le tronçon sud du périphérique dès 1985 (10). Les deux objectifs affichés alors concernent d'une part l'information aux usagers, et d'autre part fluidifier l'accès aux deux branches $A$ et $B$ de l'autoroute $A 6$ depuis la chaussée intérieure, par une meilleure répartition du trafic entre les bretelles d'accès de la porte d'Italie et de la porte d'Orléans. Les trois motivations principales affichées pour les 193 PMV finalement déployés sur le Périphérique parisien résident dans la signalisation des barrages nocturnes pour les campagnes périodiques de travaux, I'information en cas d'événement nécessitant la neutralisation d'une partie de la chaussée ou de bretelles, et enfin la communication sur les perturbations sur les six autoroutes radiales en échange direct avec le boulevard. À Lyon, le déploiement de PMV est envisagé dès le début des années 1990 mais reste réservé dans un premier temps au trafic de transit: seules les autoroutes et voies rapides urbaines sont concernées, avec une première phase de déploiement d'une trentaine de panneaux (Laborie, 1990, p. 23). Ces panneaux servent alors essentiellement à indiquer l'état du trafic sur les axes routiers traversant (autoroutes $\mathrm{A} 6$ et $\mathrm{A} 7$, axe de transit national via le tunnel de
Fourvière) ou contournant (périphérique Laurent Bonnevay, et l'autoroute A46, achevée en 1993) et les autoroutes liées, au détriment de la voirie urbaine : on retrouve bien ici les enjeux spécifiques au trafic de transit à Lyon, qui s'avèrent déterminants dans les politiques locales de gestion du trafic.

Concernant la signalisation sur la voirie urbaine, les municipalités de Paris et Lyon ont les mêmes préoccupations: des panneaux aussi lisibles et intégrés au paysage urbain que possible. Cela explique que les PMV restent peu présents en ville avant la fin des années 1990 hors voies rapides et rocades, essentiellement pour des raisons esthétiques. Ils sont intégrés par la suite dans des politiques globales de gestion de la circulation servant cet objectif de lisibilité : ainsi à Lyon, le plan de jalonnement dynamique du stationnement lyonnais de 1993 a pour premier objectif " une ville qui respire » c'est-à-dire " réduire le trafic parasite lié à la recherche de stationnement » (11). La recherche du stationnement proprement dite, "inciter l'automobiliste à se garer dans un parc » (Figure 4), n'arrive qu'en deuxième position. Par ailleurs, les indications dites « de proximité ", essentiellement statiques, font l'objet d'une politique de normalisation, dans les deux villes : les panneaux sont standardisés et les indications sont simplifiées, les municipalités veillent à leur intégration dans le paysage et le mobilier urbain existant. Cette conception, largement partagée dans les services de voirie des grandes villes, s'observe par exemple, en 1985, dans le schéma directeur de la signalisation d'indication

\section{Figure 4. Projet de panneau d'entrée de quartier pour le schéma de jalonnement dynamique des parcs de stationnement de Lyon, 1993}

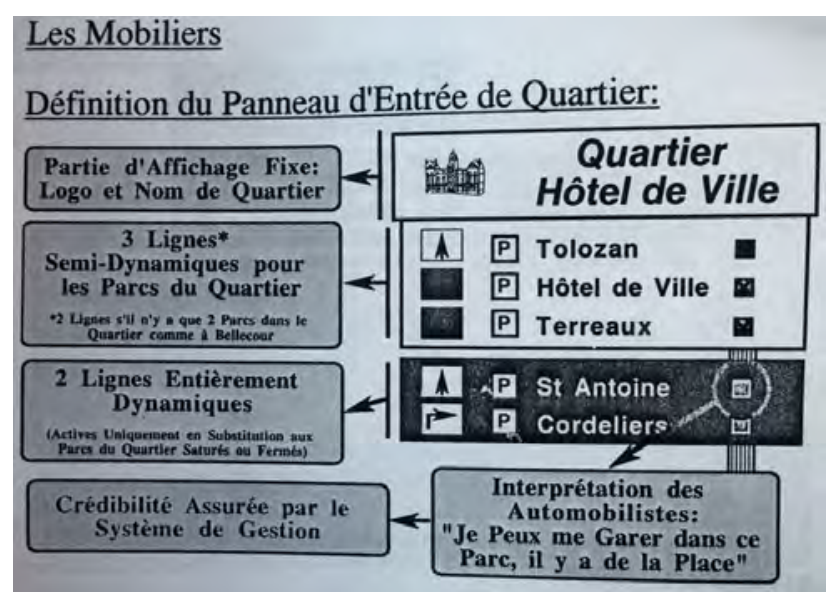

Source: AML 1781W63. 
de la Ville de Paris, sur l'étude de jalonnement de la capitale par panneaux lumineux (12): «I'accroissement du nombre des véhicules a entraîné une multiplication des panneaux réglementaires » et " la complexité croissante du tissu routier urbain a engendré la nécessité d'une signalisation directionnelle pour les véhicules afin de diminuer les temps de recherche des points de destination et de favoriser l'écoulement du trafic ». Cette recherche de normalisation et de lisibilité dans la signalisation statique montre les limites de l'empilement des dispositifs d'information aux automobilistes. Dans les deux villes, les plans de jalonnement des années 1990 prévoient des actions de simplification des panneaux : par exemple à Lyon, le nouveau schéma de jalonnement étudié en 1996 est justifié, outre
I'adaptation aux nouveaux axes routiers ouverts et au Plan de Déplacements Urbains à venir, par " la remise en cohérence de plusieurs historiques de signalisation » pour des indications plus explicites (13).

Par ailleurs, ces informations directes pour les automobilistes sont aussi destinées à être diffusées par les premiers systèmes embarqués, qui font l'objet de programmes de centralisation de l'information trafic. Dans ces programmes, le gestionnaire demeure fondamentalement la principale source des données de trafic, qui incluent à la fois les mesures du recueil automatique (capteurs dans la chaussée) et les événements (chantiers, incidents, etc.) qualifiés par les exploitants. Il en est ainsi pour le programme parisien INF-FLUX (Baudez, Georges, Henriet,

Figure 5. Schéma du projet de système de diffusion d'information CITIES

\section{SYSTEME DE DIFFUSION D' INFORMATIONS CIIIES}

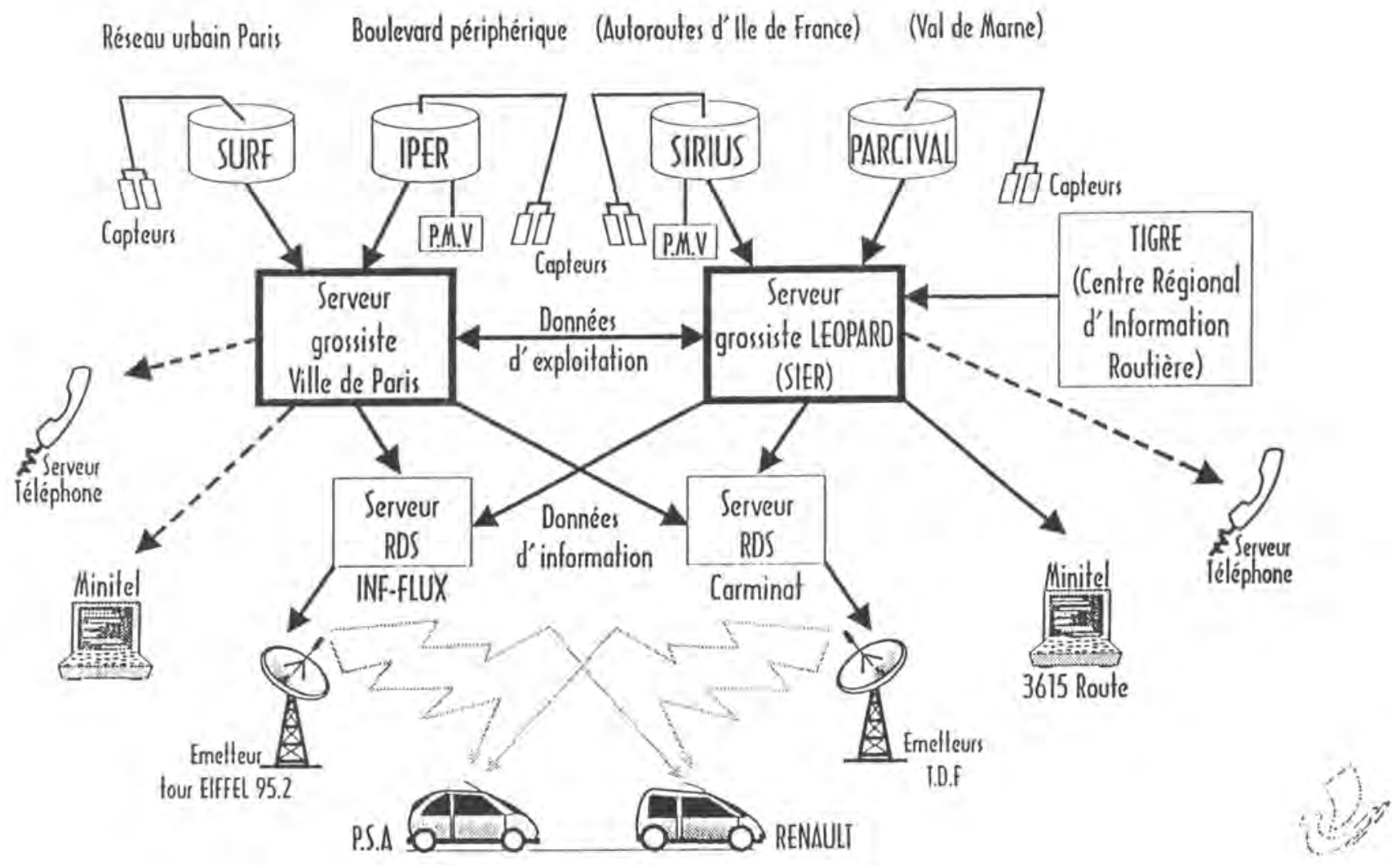

Source : Document Ville de Paris, Direction de la Voirie et des Déplacements, 1995. 
1994), dont le principe est illustré sur la Figure 5. La diffusion d'informations par ce dispositif illustre la complexité administrative et technique d'un système qui ne dépend que des capteurs et opérateurs publics, interconnectant quatre systèmes de gestion du trafic : IPER-REPER (boulevard Périphérique) et SURF (Paris intramuros) pour la Ville de Paris, SIRIUS pour les autoroutes et voies express de l'État, et PARCIVAL pour le réseau routier du Val-de-Marne. En son centre sont placés les deux serveurs grossistes de la Ville et du SIER (réseau autoroutier de I'État). Les outils producteurs de données sont placés en haut du schéma et sont pour l'essentiel constitués par les capteurs de trafic en chaussée, ainsi que les informations remontées par les opérateurs des différentes salles de contrôle. Les véhicules, cantonnés aux marques PSA et Renault (système Carminat), ne font que recevoir de l'information issue des systèmes centraux. Les fournisseurs privés de service sont absents de ce montage.

La diffusion de la télématique a donc bouleversé les politiques de régulation du trafic dans les années 1970-1980, le gestionnaire de voirie restant encore au centre des dispositifs. Au début des années 1990, les premiers terminaux embarqués sont expérimentés, sous l'égide des gestionnaires, pour une diffusion directe de l'information en temps réel aux usagers, mais semble-t-il sans modèle économique établi. II faut attendre la fin des années 1990 pour une démocratisation de l'information embarquée à bord des voitures, par des sociétés privées. Parallèlement, les gestionnaires de voirie donnent, à travers les PMV, des informations de plus en plus nombreuses et précises en temps réel.

\section{La transition vers l'usager-capteur, source d'information : un éclatement des autorités gestionnaires ?}

Le basculement de l'information trafic vers les usagers se fait de manière très progressive, sous l'effet de deux tendances communes aux deux villes étudiées. La première réside dans les évolutions technologiques qui facilitent l'accès à cette information et son appropriation par l'automobiliste. La deuxième tendance, très liée à la première, est une évolution des politiques de déplacements qui conduit les décideurs à vouloir agir sur la demande, maintenant que les dispositifs techniques permettent d'en capter la teneur: les motifs de déplacement, et le choix modal, dont la diversité est amplifiée par le développement affirmé, dans les années 1990-2000, des lignes de transport public en site propre (bus, tramway). Les années 1990 signent ainsi le début des outils publics d'information trafic locale disponibles sur Internet avec, là encore, un léger décalage chronologique entre Paris et Lyon. La capitale apparaît pionnière dans ce domaine avec un déploiement en ligne dès l'été 1996 (14), alors que Lyon doit attendre 2002 pour la mise en ligne de son système Coraly. Cela s'explique en partie par des raisons contextuelles. En région parisienne, les grèves de 1995, entraînent, en I'absence de transport public pendant plusieurs semaines, un report massif des usagers vers l'automobile et une saturation durable du réseau; cette saturation accélère le déploiement d'outils publics d'information trafic sur Internet, et marque le retour du vélo comme mode de déplacement courant.

Le premier temps du basculement vient avec l'évolution des possibilités techniques de la télématique embarquée, qui donnent lieu à différents programmes de recherche européens qui mêlent pouvoirs publics et industriels de l'automobile et des télécommunications. Parmi les plus anciens, on peut ainsi citer le programme Prometheus (Programme for European Traffic with Highest Efficiency and Unprecedented Safety), lancé en 1986, labellisé dans les projets EUREKA, et associant aux acteurs publics de la recherche, des industriels, et des constructeurs automobiles européens, parmi lesquels les français PSA et Renault. Le programme a débouché sur plusieurs prototypes en vue du guidage des véhicules dans toute l'Europe, pour des objectifs touchant à la fois à la sécurité et à l'information. On ne s'aventurera pas davantage dans l'inventaire foisonnant des projets européens des années 1980-1990 en matière de gestion du trafic (15). En résumé, l'ensemble de ces programmes a pour but une augmentation de l'efficacité des réseaux routiers, une amélioration de la sécurité routière, et une diminution des nuisances du trafic automobile : si les dispositifs sont renouvelés, les objectifs politiques restent globalement les mêmes. II est intéressant de voir que ces grands projets ont aussi donné lieu à des tentatives d'appropriations locales, aussi bien à Lyon qu'à Paris. Le programme parisien INF-FLUX mentionné plus haut s'inscrit dans le projet européen CITIES (Cooperation for Integrated Traffic Management and Information Exchange Systems) du programme DRIVE/ATT. À Lyon, le projet ULIISSE était destiné à transmettre des informations sur le trafic en temps réel à un terminal embarqué, comme on peut le voir sur la Figure 6 : le guidage vers une destination programmée était assuré en fonction des paramètres variables de la circulation (événements, surcharge des grands axes, ...). Étudié en 1989-1990, cet ancêtre de nos systèmes GPS actuels n'a pas connu de commercialisation, faute de modèle économique viable (16). Une 


\section{Figure 6. Illustration du dispositif embarqué Uliisse, Étude de faisabilité, 6 mars 1989}

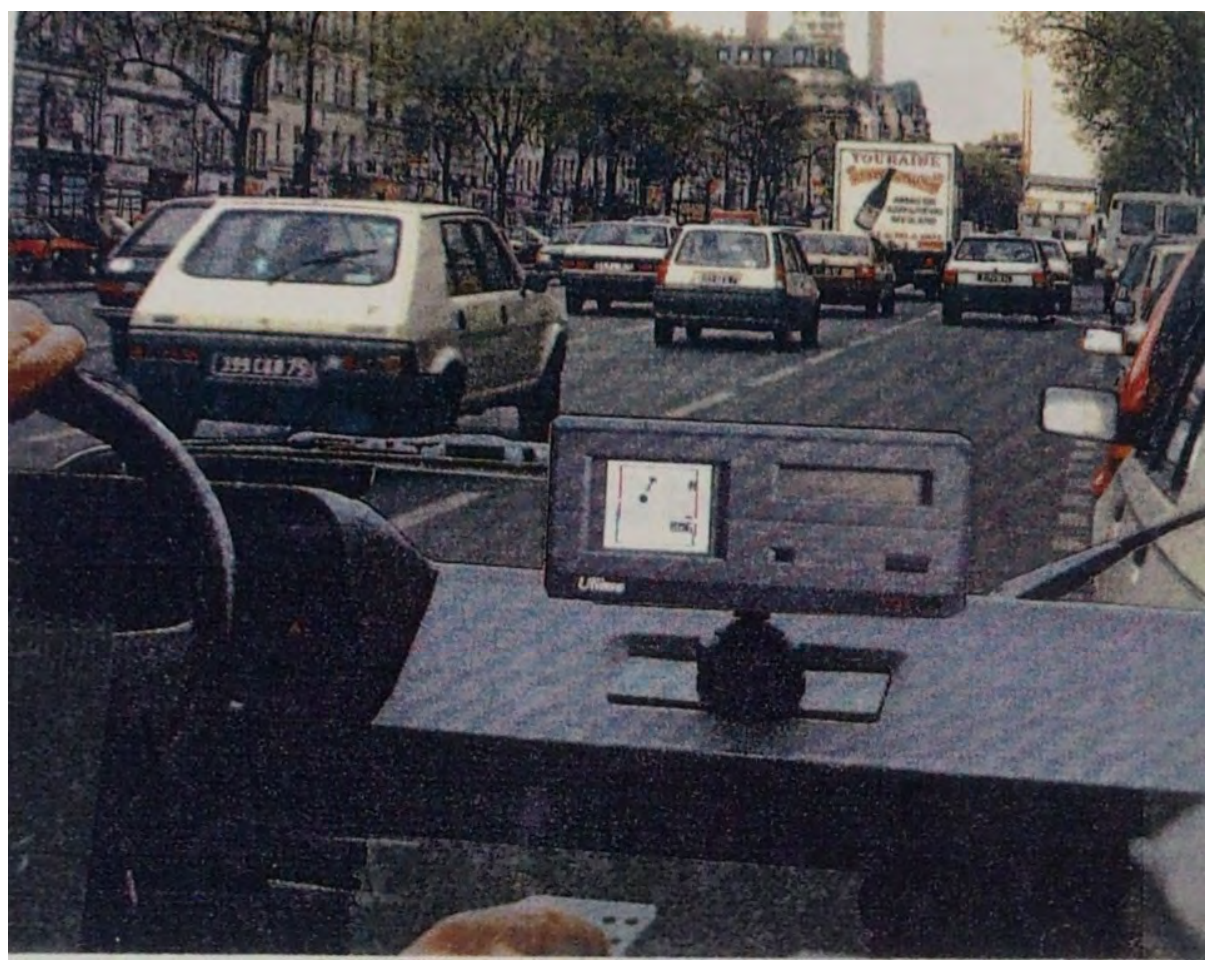

Source : Archives du Grand Lyon 2081W28

des applications majeures de ces différents programmes réside dans le déploiement en France de services de télédiffusion de I'information routière par RDS-TMC (Radio Data System/Traffic Message Channel), toujours en service.

Par ailleurs, la révolution Internet, à partir de la fin des années 1990, entraîne l'émergence de nombreux acteurs privés, qui fournissent aux automobilistes des informations à la fois basées sur les données fournies par les gestionnaires à travers des passerelles de diffusion, et progressivement, sur les données remontées par les usagers eux-mêmes. Le monopole des gestionnaires sur le contrôle et la transmission des données décline progressivement. La bascule se fait de plus en plus vers le secteur privé, qui repose encore partiellement sur l'information publique, notamment pour la crédibilité des informations événementielles (fermetures de voies, manifestations, chantiers). Cela accompagne un glissement vers le privé dans la gestion de l'information sur certaines voies : ainsi, les exploitants privés sont intégrés aux systèmes d'information trafic mis en place par les acteurs publics, dans les deux villes. Les systèmes de régulation de trafic, jusqu'alors visibles du grand public uniquement par les PMV, deviennent diffusés sur le Minitel, puis sur Internet. Le lancement en 1996 du portail « Sytadin », pour Synoptique du trafic de l'île-de-France marque l'aboutissement de l'information trafic publique francilienne, secteur également investi par le privé (lancement en 2000 du « 3615 Infotrafic » par exemple). Sytadin diffuse les données du réseau de l'État, de la Ville de Paris (réseau principal de voirie pour l'intra-muros, boulevard périphérique), et des concessionnaires autoroutiers présents en île-de-France. À Lyon, le système CORALY pour "Coordination et régulation du trafic sur les voies rapides de l'agglomération lyonnaise », suit la tendance avec un léger retard, puisque le système informatique centralisé est au point en 1998 et diffuse des informations sur le trafic sur son site Internet à partir de 2002 (17).

C'est paradoxalement alors qu'ils arrivent à maturité par leur large diffusion (PMV, Internet, téléphones mobiles) que les systèmes publics de recueil automatique de données connaissent un effondrement progressif. Cela s'observe 
davantage en île-de-France qu'en région lyonnaise, car les jeux d'acteurs sont plus complexes (notamment entre Paris et les départements qui l'entourent) et l'unité politique manque, en plus d'un vandalisme croissant (vol de câbles). Certains acteurs politiques franciliens s'émeuvent que « le plan interactif du réseau présente [...] des zones grisées correspondant aux parties du réseau non-renseignées. Ces zones sont particulièrement nombreuses, souvent stratégiques, et remettent en cause l'intérêt du système » (18). Outre les défaillances matérielles, les portails publics restent dépendants de leurs limites administratives, politiques et des gestionnaires qui ont accepté d'investir dans I'interconnexion. En Île-de-France par exemple, les systèmes de recueil des départements de petite couronne ne sont pas diffusés sur Sytadin. Cela mène l'automobiliste à la recherche de nouvelles sources de données, issues du secteur privé (Coyote, TomTom, ViaMichelin, Waze...). Parallèlement, les opérateurs privés ont repris la forme des portails publics, tout en fournissant une information plus exhaustive, y compris sur des axes que l'autorité politique ne voulait pas voir renseigner, comme certaines artères à " tranquilliser » et des voies considérées à usage exclusif de desserte locale. La situation lyonnaise semble bien moins problématique, dans la mesure où la communauté d'agglomération se distingue par une organisation institutionnelle intégrée au sein du « Grand Lyon » et où la congestion se concentre principalement sur quelques axes routiers majeurs, en dehors des voies intra-urbaines.

Ces nouveaux dispositifs accompagnent l'inflexion vers des réflexions multimodales : par exemple, les PMV affichent des temps de parcours à partir de 1994 sur le boulevard Périphérique parisien, variable commune à tous les modes de transport et attente forte des usagers, selon l'enquête de satisfaction menée alors. Afficher le temps mis en automobile est aussi vu comme une incitation au report modal des usagers, d'autant que la diffusion des temps de parcours s'étend rapidement au Minitel, aux systèmes embarqués, puis sur Internet. Une différence fondamentale semble pouvoir être faite entre Lyon et Paris sur le plan de la prise en compte de l'intermodalité sur les portails et applications d'information publique sur le trafic en temps réel. Alors que Lyon centralise les parcours possibles des principaux modes (train, métro, tramway, bus, voiture, marche, vélo) avec l'interface Onlymoov (19), qui intègre aussi les données routières de Coraly, aucun outil francilien ne combine encore, en 2017, l'automobile et les autres modes : le portail d'information public sur le trafic routier Sytadin reste centré sur l'automobile et semble étanche avec les portails multimodaux comme Vianavigo, fourni par le Syndicat des Transports d'île-de-France (aujourd'hui île-de-France Mobilités) depuis 2011. La nouvelle version de ce portail, intitulée "Tous mes déplacements en île-de-France », a été lancée en 2017, tout en omettant l'automobile des modes proposés, en dehors d'une option de "co-voiturage", et sans renvoi clair vers le portail Sytadin (20). Les orientations politiques semblent peser vis-à-vis de l'affichage des conditions de circulation automobile : la politique volontariste de report modal en faveur des transports collectifs, partagée par les deux agglomérations, n'use pas des mêmes moyens quant à l'information des usagers. L'île-de-France semble considérer que le report modal est induit par une information exhaustive sur les transports publics, sans intégrer l'automobile. De plus, la route n'est pas intégrée aux compétences régionales, et le site Vianavigo relève de la Région. Le contexte du portail lyonnais est différent, puisque I'un des enjeux du projet Optimod, dont Onlymoov est la principale interface publique, a bien été de dépasser les frontières administratives habituelles et de correspondre à un bassin de vie.

Les années 2008-2010 marquent un tournant avec l'apparition d'applications et de services numériques collaboratifs de mobilité comme Waze, racheté par Google en juin 2013. Ces réseaux reposent essentiellement sur leur base d'utilisateurs, qui fournissent en temps réel des données géolocalisées et horodatées relatives à leurs déplacements, à la marge des informations relevées et diffusées par les gestionnaires à travers leurs propres infrastructures de mesure (capteurs en chaussée, ...) et de diffusion (PMV, ...). Ils prennent une importance croissante sur le marché de l'information des automobilistes, partagé depuis la fin des années 1990 entre les gestionnaires publics et les opérateurs privés « historiques » du GPS. Waze inverse par ailleurs la tendance historique du gestionnaire seul producteur de données sur son réseau, avec le lancement en octobre 2014 du programme Connected Citizens ("Citoyens connectés »), qui vise justement à échanger des données avec les gestionnaires qui s'y affilient. Le premier but affiché est « Know what's happening on your roads », "sachez ce qu'il se passe sur vos routes » (21). Le programme marque par ailleurs un début de remise en cause du monopole public sur les installations fixes de gestion du trafic: le service Waze Beacons (balises Waze) permet l'installation, financée par le gestionnaire, de balises Bluetooth fournissant une information de localisation dans les zones non couvertes par le signal GPS (tunnels) aux utilisateurs de Waze, mais aussi aux applications compatibles (22). 
L'informatisation a ainsi permis le passage d'une information statique à un suivi dynamique et centralisé des flux automobiles par le gestionnaire, d'abord à des fins de régulation, puis pour communiquer plus directement les informations trafic aux automobilistes. Ces derniers deviennent de plus en plus des producteurs de données pour informer sur l'état du trafic mais via des entreprises privées, qui maîtrisent les données de leurs utilisateurs (production, collecte et diffusion), au détriment des logiques politiques des gestionnaires qui doivent encore parfois composer avec des frontières administratives et des logiques modales bien présentes.

\section{Conclusion}

L'information de l'automobiliste visible aujourd'hui est donc le résultat d'une stratification de dispositifs variés, qui s'explique à la fois par des évolutions technologiques importantes et par la persistance des problèmes que les politiques de gestion de la voirie ont pour but de régler : les encombrements et la sécurité des usagers. L'approche comparée de Paris et Lyon permet de constater que, si ces problèmes sont loin d'avoir la même ampleur dans les deux villes, l'évolution des objectifs politiques est globalement similaire, avec parfois un léger décalage dans le temps du côté de Lyon. Pourtant, les dispositifs mis en place connaissent une différenciation croissante : cela montre l'adaptation au contexte local et aux enjeux spécifiques de certaines politiques, comme la gestion du trafic de transit national à Lyon.

Les similitudes dans l'évolution des politiques s'expliquent en partie par une convergence dans les représentations qu'ont les gestionnaires des enjeux de l'information de l'automobiliste. Sur ce point, les évolutions entre les années 1920 et 2010 sont importantes et déterminantes dans les mutations des dispositifs. L'automobiliste à informer est d'abord considéré comme un auteur occasionnel de déplacements à diriger (tant par des règlements que des indications d'itinéraires). À partir des années 1950, il est vu comme une partie de flux en voie de massification, donc à réguler, avant d'être reconsidéré de manière plus individuelle à partir des années 1990 comme demandeur d'informations précises, en temps réel sur son déplacement. L'évolution est aussi perceptible dans les pratiques de gestion des voies urbaines, dont l'information de l'automobiliste constitue un instrument parmi d'autres : cette gestion devient de plus en plus globale, avec des plans à l'échelle de la ville entière et une volonté de centralisation des dispositifs qui passe notamment par la normalisation des panneaux et la centralisation de la régulation dans des postes de contrôle (PC) de la circulation. La signalisation routière statique est longtemps restée dominante, avant le développement des dispositifs dynamiques ou embarqués à partir des années 1980. Cela est permis par des innovations technologiques, plus particulièrement l'informatisation, mais l'accès à l'information dépend avant tout de choix politiques de gouvernance du trafic.

En outre, I'ambition globalisante des politiques d'information de l'automobiliste se manifeste paradoxalement par une prise en compte croissante du caractère individuel du déplacement, à partir des années 1970, dans le cadre de politiques plus larges que la seule gestion de la circulation: la gouvernance de la voirie passe de plus en plus par des politiques multimodales, à accompagner par des dispositifs d'information complexes qui impliquent des acteurs de plus en plus nombreux. La coordination des acteurs et des politiques publiques reste un enjeu important de l'information à l'automobiliste et de ses dispositifs : I'unité politique plus affirmée à Lyon qu'à Paris est un élément déterminant de la différenciation des dispositifs d'information embarqués développés à partir des années 1990. Par ailleurs, la rigidité des cadres institutionnels et des découpages territoriaux desservent les gestionnaires publics par rapport à des entreprises privées qui tendent, ces dernières années, à prendre le pas sur l'information trafic publique : mettant l'automobiliste au centre des dispositifs, elles en font aussi un producteur de données, brisant ainsi le monopole des informations trafic des gestionnaires publics. De fait, les données de trafic ne sont plus produites uniquement par les gestionnaires de la voirie, mais par les véhicules et usagers y circulant. Les entreprises privées sont ainsi en mesure d'informer exhaustivement, sur l'ensemble des réseaux et des voies, leurs clients automobilistes, indépendamment des gestionnaires publics.

Comme l'attestent les actuels débats sur l'ouverture, en Open Data, des données de mobilité, la gouvernance des transports et de ses données pose toujours la question de la place du secteur privé dans l'information des usagers. Les problématiques de divergence d'intérêts tant politiques qu'économiques, entre gestionnaires publics et entreprises privées, mériteraient d'être approfondies.

Louis Baldasseroni est doctorant en Histoire contemporaine à I'Université Paris-Est, Laboratoire ACP/Labex Futurs Urbains. 
Sa thèse s'intitule : "Du macadam au patrimoine : modernisation de la voirie et conflits d'usage, l'exemple de Lyon, fin XIXe - fin XXe siècle ».

louis.baldasseroni@univ-paris-est.fr
Luc Charansonney est docteur en Transports et a réalisé sa thèse à l'Université Paris-Est, au LVMT (UMR T 9403) (Laboratoire Ville, Mobilité, Transport) École des Ponts ParisTech, IFSTTAR, UPEMLV. Il est Ingénieur des Travaux de la Ville de Paris et travaille à la Direction de la Voirie et des Déplacements, Ville de Paris. luc.charansonney@paris.fr

\section{Notes}

(1) AML (Archives Municipales de Lyon) 1127WP60, Avant-projet du plan de signalisation de Lyon, 23 mai 1931.

(2) AML 1068WP1, Règlement général de la circulation de Lyon, Imprimerie Lyonnaise, 1926.

(3) AML 1127WP60, Avant-projet du plan de signalisation de Lyon, 23 mai 1931.

(4) De I'anglais traffic engineering, née dans les années 1920 aux États-Unis (Eno Foundation), puis se diffusant progressivement en Europe (Royaume Uni, France).

(5) Voir par exemple : Adams, 1936.

(6) Mairie de Paris, Direction de la Voirie, Service Technique des Études, Schéma directeur de la signalisation d'indication, 1985.

(7) Mairie de Paris, Direction de la Voirie, Service Technique des Études, Schéma directeur de la signalisation d'indication, 1985.

(8) AGL (Archives du Grand Lyon) 1973WM1-1, Délibération du Conseil de Communauté Urbaine, 17 novembre 1980.

(9) AML 2385WP2, Note sur l'organisation du programme Leader, COURLY, 1992.

(10) Bloch Jean-Pierre, Présentation du système d'information des usagers du Corridor Périphérique par panneaux à messages variables, Mairie de Paris, Mai 1985

(11) AML 1781WP63, Avant-projet du plan de jalonnement dynamique du stationnement à Lyon, 1993.

(12) Mairie de Paris, Direction de la Voirie, Service Technique des Études, Schéma directeur de la signalisation d'indication, 1985.

(13) AML 1795W27, Document de travail sur le Plan de jalonnement de l'agglomération lyonnaise, 19 août 1996.
(14) « Si ce lancement [celui de Sytadin, NDA] n'en est pas la conséquence directe, il a été sinon motivé, du moins accéléré par les perturbations engendrées par les mouvements de grèves de I'hiver 1995. ", DIR Île-de-France, Sytadin, Un peu d'histoire, 1996.

(15) Pour cela, voir : Lannoy, 2003.

(16) AGL 2081W28, Projet Uliisse, Étude de faisabilité, 6 mars 1989.

(17) «Découvrez Coraly » [En ligne] (Page consultée le 8 juin 2017) Disponible à l'adresse : http://www.coraly.com/ Decouvrez-Coraly/Historique

(18) Question orale sans débat $n^{\circ} 05335$ de Mme Catherine Procaccia (Val-de-Marne - UMP) publiée dans le JO du Sénat du 01/08/2013 - page 2228 [En ligne] (Page consultée le 8 juin 2017) Disponible à l'adresse : http://www.senat.fr/questions/base/2013/qSEQ13080533S.html

(19) Page d'accueil du site Internet Onlymoov [En ligne] (Page consultée le 8 juin 2017) Disponible à l'adresse: http://www.onlymoov.com

(20) Page d'accueil du site Internet Vianavigo [En ligne] (Page consultée le 8 juin 2017) Disponible à l'adresse: http://lab.vianavigo.com/accueil

(21) Page d'accueil du site Internet Waze [En ligne] (Page consultée le 8 juin 2017) Disponible à l'adresse : https://www. waze.com/ccp

(22) Waze Beacons Program, «Améliorons la navigation dans les tunnels des villes du monde entier» [En ligne] (Page consultée le 8 juin 2017) Disponible à l'adresse : https://www. waze.com/fr/beacons

\section{Bibliographie}

Adams W.F., 1936, Road Traffic Considered as a Random Series, Journal of the Institution of Civil Engineers, Vol. 4, Issue 1, p. 121-130.

BANNAT F., 1995, La suppression des tramways à Paris ", L'histoire de la Rue, journée organisée par la Direction de la Voirie, Mairie de Paris, Direction de la Voirie.

Barles S., Guillerme A., 1998, La congestion urbaine en France (1800-1970) [En ligne] (consultée le 23 mai 2017) Disponible à l'adresse : https://halshs.archives-ouvertes.fr/ halshs-00280970

Baudez G., Georges P., Henriet A., 1994, Mise en œuvre du concept Inf-Flux(R), Transports, Environnement, Circulation, $\mathrm{n}^{\circ} 126$, p. $40-43$.
Debizet G., 2011, L'évolution de la modélisation des déplacements urbains en France 1960-2005, Flux, 2011/3-4 $\left(n^{\circ} 85-86\right)$, p. 8-21.

Duhamel-Herz M., Nouvier J., 1998, La signalisation routière en France de 1946 à nos jours, Paris : AMC.

Dupur G., 1975, Une technique de planification au service de l'automobile, les modèles de trafic urbain, Paris : Ministère de l'Équipement.

Faugier E., 2009, Automobile, transports urbains et mutations : I'automobilisation urbaine de Québec, 1919-1939, Urban History Review, Vol. 38, n 1, p. 26-37.

Flonneau M., 2003, L'automobile à la conquête de Paris, Paris : Presses de l'École nationale des Ponts et Chaussées. 
Flonneau M., 2005, Paris et l'automobile : un siècle de passions, Paris : Hachette.

GaRdON S., 2009, Gouverner la circulation urbaine : des villes françaises face à l'automobile : années dix-années soixante, thèse de Sciences Politiques sous la direction de Gilles Pollet, Université Lyon II [en ligne] (consulté le 27 mai 2017) Disponible à l'adresse: http://theses.univ-lyon2.fr/ documents/lyon2/2009/gardon_s\#p=0\&a=top

Gardon S., 2011, Goût de bouchons : Lyon, les villes françaises et l'équation automobile, Paris : Descartes \& Cie.

Greenshields B. (ed), 1947, Traffic performance at urban street intersections, Bureau of Highway Traffic, Technical Report No. 1.

\section{Résumé - Louis Baldasseroni, Luc Charansonney - Gouverner la voirie urbaine par l'information de I'automobiliste. Une comparaison Lyon-Paris, des années 1920 à nos jours}

L'information des automobilistes est un élément-clé des politiques de gestion des voies urbaines. L'analyse comparée des cas de Lyon et Paris entre les années 1920 et 2010 montre que les dispositifs actuels d'information aux automobilistes résultent de l'empilement de trois strates politiques et techniques. Au cours de la période, Paris et Lyon suivent sensiblement les mêmes orientations politiques. Cependant, Paris ne constitue pas un modèle pour Lyon : I'autonomie locale conduit à des réponses techniques différentes aux problèmes de gestion de la circulation. Des années 1920 aux années 1940, I'automobiliste est vu par le gestionnaire comme un individu qui doit être guidé et discipliné, surtout par des panneaux de signalisation statiques. Par la suite, des années 1950 aux années 1970, les autorités gèrent des flux plus que des individus. Des systèmes centraux, reposant sur des capteurs dans la chaussée, et dédiés aux gestionnaires, sont mis en place en parallèle $d^{\prime}$ une politique d'infrastructures nouvelles. Suite au choc pétrolier, I'information aux automobilistes devient multimodale. Le conducteur est de nouveau vu comme un individu se déplaçant et en quête d'information. Dans les années 1990, le gestionnaire fournit une information centralisée à l'automobiliste, par le biais des panneaux à message variable et sites internet publics. Parallèlement, les systèmes embarqués permettent à des entreprises privées de collecter des données de trafic et de fournir de l'information directement à leurs clients-automobilistes, sans passer par le gestionnaire. Cela influe sur les rapports public-privé dans la gouvernance du trafic.

Mots-clés : information routière, gestion du trafic, signalisation, histoire urbaine, histoire des routes
Laborie P., 1990, Coraly, Transports, Environnement, Circulation, $\mathrm{n}^{\circ} 101$, p. 46-48.

Lannor P., 2003, Le problème de la circulation et la promesse télématique, Paris : Publibook.

MONTÈs C., 2003, Les transports dans l'aménagement urbain à Lyon, Lyon : Géocarrefour.

Rosenthal J., 2006, L'information routière au service de I'usager: de Bison Futé au schéma directeur d'information routière, Transports, Environnement, Circulation, n 188 , p. 26-30.

\section{Abstract - Louis Baldasseroni, Luc Charansonney - Governing urban roads by informing drivers: comparing Paris and Lyon from the 1920s onwards}

Informing car drivers is a cornerstone of policies of urban road governance. Through comparative analysis of Paris and Lyon from 1920 to 2010, we show that today's driver information results from three technical and political strata. Through the period, Paris and Lyon basically followed the same traffic policies. Nonetheless Paris did not always act as a model for Lyon as the local context led to different technical responses to traffic management needs. From the 1920s to the 1940s, the driver was seen as an individual, to whom the road authorities needed to provide regulation and guidance, mainly through static signposting. Then, from the 1950s to the late 1970s, the authorities dealt with traffic flows rather than individuals. Central systems, relying on road sensors and dedicated to the authorities in charge, were developed along with new infrastructures. Following the oil crisis, driver information became multi-modal. The driver was once again an individual travelling and in need of information. By the 1990s, authorities provided centralized traffic information on variable message signs and on the Internet. Simultaneously, on-board devices allowed private companies to gather traffic data and provide traffic information to their driver-clients, independently of the road authorities. This has had consequences on how public and private actors govern traffic with diverging interests.

Keywords: driver information, traffic management, roadsigns, urban history, road history, french cities 